\title{
PREFACE
}

Small RNA pathways or RNA silencing is a group of pathways, which utilize small (20$30 \mathrm{nt}$ ) RNAs as guides for sequence-specific repression. This collection of texts on small RNA pathways originates from a report I prepared for the European Food and Safety Authority (EFSA) in 2016 and 2017. EFSA requested literature survey as it was concerned because of several works reporting that small RNAs, which naturally exist in plants, can enter into a mammalian body and affect gene expression. The main issue was that RNA interference (one of a small RNA pathways), represented a promising way for developing sequence-specific pesticides. If plant small RNAs could enter into a mammalian organism and regulate genes, RNAi-based pesticides could represent a potential health hazard.

With help of my colleagues, who set up a systematic literature search, I wrote an extensive systematic literature review for which I inspected $~ 10,000$ titles and abstracts during one year of writing. The original EFSA report had a number of technical sections describing search methodology and was compiling information from published works for specific tasks defined by EFSA. Once the report was completed and presented to EFSA, I became interested in converting the scientific part of the report into a more coherent overview of RNA silencing (particularly of RNAi and microRNA pathways) across different animal groups and plants. I thought it would be better if the scientific part would be revised, and provided as a coherent collection of chapters for studying RNAi and related pathways.

I subsequently contacted EFSA and discussed possible options for producing a set of chapters based on the report, which could serve as a study material for my lecturing. EFSA representatives agreed that I could produce a set of study materials from the report that would be published by a university publishing house under conditions that EFSA would be credited and the book would not be sold - as a solution, the material is provided as an open access collection of chapters. To satisfy the second EFSA requirement, I acknowledge hereby that the contents have been produced under a contract with EFSA (OC/EFSA/ GMO/2015/01-CT 01) and that the opinions expressed are those of the contractor only and do not represent EFSA's official position.

Regarding the conversion, the original text was reorganized into twelve chapters, which were reformatted and revised in order to remove copyrighted material from third parties and provide a introductory parts for stand alone chapters. Nine of the chapters focus on small RNA pathways in animals (mammals, birds, fish, arthropods, nematodes, molluscs, and annelids) and plants. The remaining three chapters include a general introduction and reviews of important phenomena - off-targeting and extracellular small RNAs. I hope that this collection will serve as a useful source for many.

\section{Petr Svoboda}

